\title{
Aerosol-Cloud Interactions In Global Models Of Indirect Aerosol Radiative Forcing
}

\author{
Athanasios Nenes and John H. Seinfeld \\ Department of Chemical Engineering, California Institute of Technology, Pasadena, CA, 91125
}

\begin{abstract}
The sensitivity of cloud optical properties with respect to parameters that affect aerosol activation is examined. Of particular interest are the effect of volatile gases (such as $\mathrm{HNO}_{3}$ ), slightly soluble and surfactant species. An adiabatic parcel model is used to simulate cloud droplet formation. Cloud optical properties are calculated from these simulations.
\end{abstract}

\section{INTRODUCTION}

Clouds are one of the key aspects of the climate system and have to be included in any climate simulation. However, computational constraints prohibit a comprehensive description of cloud formation in global climate models; instead, clouds are incorporated through simplistic parameterizations. As a result, the uncertainty introduced may be sufficiently large to rival the anthropogenic component of climate forcing. Much of this uncertainty originates in the complex relationship between cloud droplets and the aerosols they originate from. Both experimental and approximate analytical expressions that relate aerosol particles to cloud droplets have been proposed for implementation in climate models ${ }^{1}$. Idealized assumptions regarding the aerosol composition and cloud dynamics tend to limit the applicability of these relations. Furthermore, effects that can significantly alter the activation behavior of the aerosol, such as the presence of semi-volatile gases (e.g., $\mathrm{HNO}_{3}$ ), slightly soluble ${ }^{2}$, and surfactant species ${ }^{3}$ are not accounted for. Errors in cloud droplet number become particularly important when cloud radiative properties are calculated, since they can be very sensitive to changes in droplet number ${ }^{4}$.

This work attempts to determine which of the parameters affecting aerosol activation potentially has the largest effect on cloud optical properties, elucidating the parameters that should be included in a global aerosol-cloud droplet parameterization. For the purposes of this study, an adiabatic cloud parcel model with explicit aerosol microphysics is used to simulate the growth of $\mathrm{CCN}$ to cloud droplets within a cloud. From the simulations, cloud optical properties are calculated, and the sensitivity of the effective radius, optical thickness, and cloud albedo is computed with respect to the parameters considered. 


\section{CLOUD PARCEL AND RADIATIVE MODELS}

A cloud parcel model is the simplest tool that can be used to simulate the evolution of droplet distributions throughout a non-precipitating convective cloud column. Although highly idealized, these models offer the most computationally efficient way to explore parametric space.

\section{Cloud Parcel Model}

The parcel model used is described in [5, 6]. In this model, conservation of heat and moisture are written for a rising air parcel. Moisture from the vapor phase is depleted by the aerosol population existing within the parcel. This depletion rate depends on the growth rate of each aerosol particle ${ }^{6}$ which is primarily due to the condensation of water vapor on the particle,

$$
\frac{d r}{d t} \propto \frac{\left(S-S_{e q}\right)}{r}
$$

where $r$ is the particle size and $S$ is the local saturation ratio. The equilibrium saturation ratio $S_{e q}$ is equal to

$$
S_{e q}=\exp \left(\frac{2 \sigma}{R T v r}-\frac{i_{s} n_{s}}{n_{w}}-\frac{i_{v} n_{v}}{n_{w}}-\frac{i_{s s} n_{s s}}{n_{w}}\right)
$$

where $v, \sigma, \rho$ are the molar volume, surface tension, and density of the droplet, respectively, $R$ is the universal gas constant, $T$ is the temperature, and $n_{w}, n_{s}, n_{v}, n_{s s}$ are the number moles of water, soluble salt, volatiles, and slightly soluble species in the droplet. Finally, $i_{s}, i_{v}, i_{s s}$ are the van't Hoff factors for the soluble salt, volatile species, and slightly soluble species dissolved within the droplet.

Equation 2 incorporates the effects considered in this study that influence aerosol activation. The first term in the exponential is the Kelvin effect, while the remaining terms express the solute, volatiles and slightly soluble effects, respectively.

In addition to water vapor, transfer of volatiles between the particles and gas phase takes place:

$$
\frac{d n_{v}}{d t} \propto\left(P_{v}-P_{v, e q}\right)
$$

where $P_{v}$ is the partial pressure of the volatiles in the gas phase, and $P_{v, e q}$ is the equilibrium partial pressure of the volatiles over a given particle, and is calculated by a thermodynamic equilibrium module.

\section{Cloud Albedo}

Cloud albedo, $R_{c}$, is calculated based on the two-stream approximation of a nonabsorbing, horizontally homogeneous cloud ${ }^{6}, R_{c}=\frac{\tau}{7.7+\tau}$, where $\tau$ is the cloud optical depth, $\tau=\int_{0}^{H} \frac{3 \rho_{a} w_{L}(z)}{2 \rho_{w} r_{e f f}(z)} d z$, and $w_{L}(z)$ is the liquid water mixing ratio profile 
along the cloud column, calculated from the parcel model simulations. In addition, $\rho_{w}$ is the water density, $\rho_{a}$ is the air density, $z$ is the cloud depth, and $r_{\text {eff }}(z)$ is the cloud droplet distribution effective radius.

\section{SIMULATION PARAMETERS}

Cloud optical properties depend on cloud thickness, cooling rate, volatile gas concentration, and aerosol size distribution characteristics. For each parameter, we consider values that cover the range found in experimental observations.

\section{Cloud Parameters}

Cloud thickness affects the transit time of air parcels within the cloud, and hence the time available for particle growth. We consider values ranging from 10 to $1000 \mathrm{~m}$, which covers the thickness range seen for boundary layer clouds ${ }^{7}$.

Updraft velocity influences both the transit time and the maximum supersaturation in a cloud updraft. Observed updraft velocities in boundary layer clouds vary widely, but values derived from measured vertical velocity variance are typically ${ }^{7}$ between 30 and $50 \mathrm{~cm} \mathrm{~s}^{-1}$. We explore the dependence on updraft by considering updraft velocities of $10,30,100$, and $300 \mathrm{~cm} \mathrm{~s}^{-1}$.

Finally, the volatile species considered in this study is $\mathrm{HNO}_{3}$, and is assumed to have an initial mixing ratio ranging between 0 and $5 \mathrm{ppb}$.

\section{Aerosol Parameters}

The sensitivity of cloud optical properties with respect to aerosol distribution parameters will be explored by considering two aerosol size distributions, one representing pristine and the other polluted conditions. For this purpose, we use the marine (pristine) and urban (polluted) trimodal log-normal size distributions of [8]. The aerosol number concentration, $N_{i}$, the number mode radius, $r_{g, i}$, and geometric standard deviation, $\sigma_{i}$, for each aerosol type are listed in Table 1.

\begin{tabular}{lccc}
\multicolumn{4}{l}{ TABLE 1. Aerosol distribution parameters $\left(\boldsymbol{r}_{g, i}\right.$ in $\mu \mathbf{m}, \boldsymbol{N}_{i}$ in $\left.\mathbf{~ c m}^{-\mathbf{3}}\right)$} \\
\hline Aerosol type & Nuclei mode & Accumulation mode & Coarse mode \\
& $\left(r_{g, 1}, \sigma_{1}, N_{1}\right)$ & $\left(r_{g, 1}, \sigma_{1}, N_{1}\right)$ & $\left(r_{g, 1}, \sigma_{1}, N_{1}\right)$ \\
\hline Marine & $0.005,1.6,340$ & $0.035,2.0,60$ & $0.31,2.7,3.1$ \\
Urban & $0.007,1.8,106000$ & $0.027,2.16,32000$ & $0.43,2.21,5.4$ \\
\hline
\end{tabular}

In each of the distributions examined, the partially soluble mass fraction is allowed to vary between $0 \%$ and $50 \%$. The solubility of the partially soluble fraction is also allowed to vary between $10^{-1}$ to $10^{-4}$ moles $I^{-1}$. The soluble fraction is assumed to be composed of $\left(\mathrm{NH}_{4}\right)_{2} \mathrm{SO}_{4}$, while the slightly soluble fraction is an organic with molar mass of $0.176 \mathrm{~kg} \mathrm{~mol}^{-1}$ and a van't Hoff factor equal to 2 . When the effect of surface tension is considered, the following experimental relation ${ }^{9}$ is used: 


$$
\frac{\sigma-\sigma_{w}}{T}=1.87 \times 10^{-5} \ln \left(1+628.14 C_{s s}\right)
$$

where $C_{s s}$ is the concentration of slightly soluble species (moles $\mathrm{l}^{-1}$ ), and $\sigma_{w}$ is the surface tension of pure water $\left(\mathrm{N} \mathrm{m}^{-1}\right)$.

\section{SIMULATION RESUTS-DISCUSSION}

The "base case" simulation refers to an aerosol composed exclusively of soluble salt, without the presence of volatiles. Figure 1 presents the maximum albedo difference throughout the cloud column with respect to the base case simulation (marine aerosol), when various effects in equation (2) are included. The effect of slightly soluble species does not seem to significantly alter the cloud albedo. However, including surface tension changes can potentially play an important role for large updrafts. This effect is sensitive to the surfactant content of the aerosol, which tends to be less than $10 \%$ in pristine environments. In addition, the simulations show that solubility of the surfactant affects the maximum albedo difference between the range of $10^{-4}$ to $10^{-3}$ moles $l^{-1}$. Finally, volatiles can have a significant effect, being less sensitive to changes in updraft velocity.

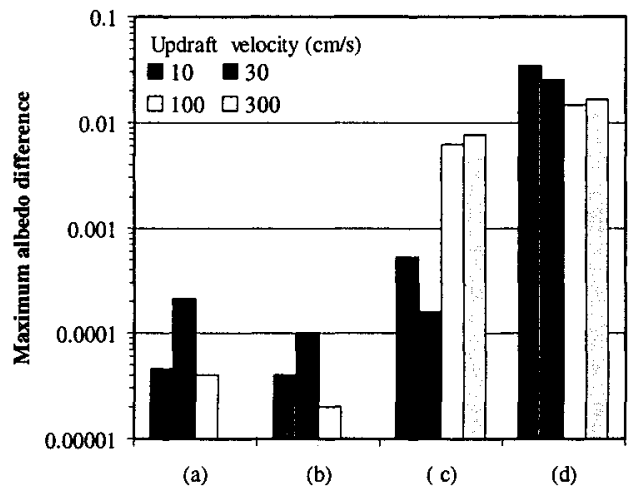

\section{REFERENCES}

1. Abdul-Razzak, H., Ghan, S. J., and Rivera-Carpio, C., J. Geophys. Res., 103, 6123-6132 (1998).

2. Laaksonen, A., Korhonen, P., Kulmala, M. and Charlson, R. J., J.Atmos.Sci., 55, 853-862 (1998).

3. Shulman, M. L., Jacobson, M. C., Charlson, R. J., Synovec, R. E., and Youngs, T. E., Geophys.Res.Letters, 23, 277-280 (1996).

4. Pincus, R., and Baker, M., Nature, 372, 250-252 (1994).

5. Seinfeld, J.H., and Pandis, S. N., Atmospheric Chemistry and Physics, New York: John Wiley (1998).

6. Nenes, A., Ghan, S., Abdul-Razzak, H., Chuang, P. Y., and Seinfeld, J.H., submitted to Tellus $(2000)$

7. Nichols, S., Quart J. R. Met. Soc., 110, 783-820 (1984)

8. Whitby, K.T., Atmos. Environ., 12, 135-159 (1978)

9. Facchini M. C., Mircea M., Fuzzi S., Charlson R. J., Nature, 401, 257-259 (1999) 